\title{
MicroRNA-I48b suppresses proliferation, migration, and invasion of nasopharyngeal carcinoma cells by targeting metastasis-associated gene 2
}

This article was published in the following Dove Press journal:

OncoTargets and Therapy

2 June 2017

Number of times this article has been viewed

\author{
Minhua $\mathrm{Wu}^{1, *}$ \\ Xiaoxia Ye ${ }^{1, *}$ \\ Shengchun Wang ${ }^{2, *}$ \\ Qinghua $\mathrm{Li}^{3}$ \\ Yinxuan $\mathrm{Lai}^{4}$ \\ Yanmei $\mathrm{Yi}^{\prime}$ \\ 'Department of Histology and \\ Embryology, Guangdong Medical \\ University, Zhanjiang, People's \\ Republic of China; ${ }^{2}$ Department \\ of Pathology, Guangdong Medical \\ University, Dongguan, People's \\ Republic of China; ${ }^{3}$ Department \\ of Hematology, Affiliated Hospital \\ of Guangdong Medical University, \\ Zhanjiang, People's Republic of \\ China; ${ }^{4}$ Health Management and \\ Medical Examination Center, \\ Affiliated Hospital of Guangdong \\ Medical University, Zhanjiang, \\ People's Republic of China \\ *These authors contributed equally \\ to this work
}

Purpose: MicroRNAs (miRNAs) play important roles in tumorigenesis and metastasis by regulating genes expression. MiRNA-148b (miR-148b) had been reported to inhibit tumor progression in some kinds of cancers, but the functions of miR-148b in nasopharyngeal carcinoma (NPC) remain largely unknown. The aim of this study was to investigate the functional role of miR-148b in NPC.

Methods: Expression of miR-148b in NPC tissues and cell lines was detected by quantitative reverse transcription polymerase chain reaction. MiR-148b was overexpressed in CNE2 and C666-1 cells by miR-148b mimic transfection. The effects of miR-148b on cell proliferation, migration, and invasion were determined by colony formation assays, cell viability assays, and transwell assays. The target gene of miR-148b was investigated by luciferase assays, and the rescue experiment was performed.

Results: MiR-148b was downregulated in NPC tissues and cell lines. Ectopic miR-148b expression significantly inhibited proliferation, migration, and invasion of CNE2 and C666-1 cells. We identified that metastasis-associated gene 2 (MTA2) is a direct target of miR-148b. Rescue experiment demonstrated that the tumor-suppressive effects of miR-148b on C666-1 cell were partly reversed by restoration of MTA2 expression. Moreover, miR-148b expression was negatively related to mRNA level of MTA2 in NPC tissues.

Conclusion: Our findings elucidate that miR-148b negatively regulates the growth, migration, and invasion of NPC cells, at least in part, by targeting MTA2. The present study indicates that miR-148b is a potential therapeutic agent for NPC.

Keywords: miR-148b, metastasis-associated gene 2, proliferation, invasion, nasopharyngeal carcinoma

\section{Introduction}

Nasopharyngeal carcinoma (NPC), a unique type of the head and neck cancer, is most prevalent in Southeast Asia and Southern China. In some regions of these endemic areas, the incidence rate of NPC is more than 10/100,000. However, the incidence rate of NPC in non-endemic areas is less than $1 / 100,000 .{ }^{1}$ Radiation therapy is effective in the treatment of NPC, especially for early-stage NPC patients. However, a large proportion of NPC patients are diagnosed at advanced stage. Each year in China, about 20,000 people die of NPC, mainly due to tumor recurrence and metastasis. ${ }^{2}$ It is of great urgency to elucidate the molecular mechanisms of metastasis in NPC, which is critical for identifying new biomarkers and treatment strategies.

MicroRNAs (miRNAs) are endogenous small noncoding RNA, which can regulate genes expression by binding to the specific recognition sequences in the $3^{\prime}$-untranslated
Correspondence: Yanmei Yi Department of Histology and Embryology, Guangdong Medical University, 2 Wenming East Road, Zhanjiang, Guangdong 524023, People's Republic of China Email chumeng77@I63.com 
region (UTR) of target mRNAs. ${ }^{3}$ MiRNAs have been shown to play important roles in tumorigenesis, progression and metastasis in NPC and other cancers by regulating the expression of target genes. ${ }^{4,5}$ Exploration of dysregulated miRNAs in NPC might contribute to the discovery of new biomarkers valuable for diagnosis or therapy of NPC.

MiRNA-148b (miR-148b) had been reported to be downregulated, and it acts as a tumor suppressor in human lung cancer, ${ }^{6}$ glioma, ${ }^{7}$ pancreatic cancer ${ }^{8}$ and hepatocellular carcinoma. ${ }^{9}$ However, miR-148b is found to be upregulated in human ovarian carcinoma. ${ }^{10}$ In normal Schwann cells, the upregulation of miR-148b promoted cell migration. ${ }^{11}$ These studies suggest that miR-148b may play different roles in different situations.

To date, the functional role of miR-148b in NPC is still largely unknown. In this study, we found that miR-148b was downregulated in NPC tissues and cell lines. Ectopic miR-148b expression significantly inhibited proliferation, migration, and invasion of NPC cells. Metastasis-associated gene 2 (MTA2), which is associated with malignant behaviors of tumor cells, ${ }^{12}$ is identified to be a direct target of miR-148b. Our findings demonstrated, for the first time, that miR-148b could suppress NPC cell growth, migration, and invasion by targeting MTA2.

\section{Materials and methods Patients and tissue samples}

Samples of 20 noncancerous nasopharyngeal tissues and 25 NPC tissues were obtained from the Affiliated Hospital of Guangdong Medical University, Zhanjiang, China, between 2013 and 2014. The use of the clinical materials was approved by the medical ethical committee of the hospital. All the patients enrolled in this study had signed the informed consent documents. The NPC patients had not received any radiotherapy or chemotherapy before biopsy. The patients comprise 17 men and 8 women with an average age of 45.7 years (range, 22-70 years). The clinical stage of patients was based on the pathology tumor-node-metastasis (pTNM) system issued by American Joint Committee on Cancer/Union for International Cancer Control in 2002 (stage I: one case; stage II: six cases; stage III: 16 cases; stage IV: three cases). All samples of NPC patients were diagnosed to be nasopharyngeal nonkeratinizing carcinoma.

\section{Cell culture}

Four human NPC cell lines (CNE1, CNE2, C666-1 and HNE1) and an immortalized nasopharyngeal epithelium cell, NP69, were obtained from the Cancer Research Institute of
Guangdong Medical University. The application of these cell lines in this study was approved by the Medical Ethics Committee of Guangdong Medical University. NPC cells were maintained in Dulbecco's Modified Eagle's Medium (DMEM) containing 10\% fetal bovine serum (FBS). NP69 cell was cultured in keratinocyte serum-free medium (Thermo Fisher Scientific, Waltham, MA, USA). All the cells were grown in a humidified atmosphere at $37^{\circ} \mathrm{C}$ with $5 \% \mathrm{CO}_{2}$.

\section{RNA isolation and quantitative reverse transcription polymerase chain reaction (qRT-PCR)}

Total RNA of cells or tissues was extracted using TRIzol reagent (Thermo Fisher Scientific). The cDNA was synthesized using the PrimeScript RT Reagent Kit (Takara, Dalian, China). For the detection of miR-148b level, miRNAs were reversely transcribed using the One Step PrimeScript miRNA cDNA Synthesis Kit (Takara) and the amplification processes were performed using the SYBR Premix Ex Taq II Kit (Takara) by a Light Cycler machine (Hoffman-La Roche Ltd, Basel, Switzerland). The primers for miR-148b and U6 small nuclear RNA (snRNA) were purchased from Ambion (Thermo Fisher Scientific). U6 snRNA was used as an internal control of miR-148b. For the detection of MTA2 mRNA level, the cDNA was synthesized using the PrimeScript RT Reagent Kit (Takara) and then amplified using an SYBR Green PCR Kit (Takara). The primers for MTA2 and $\beta$-actin were described previously, ${ }^{13}$ and $\beta$-actin was used as an internal control of MTA2. The PCR results were normalized to internal controls and analyzed according to the $2^{-\Delta \Delta \mathrm{Ct}}$ method. Three independent experiments were performed.

\section{Transfection of miRNA mimic and MTA2 expression plasmid}

MiR-148b mimic or control RNA (miR-Ctrl) were designed and synthesized by RiboBio (Guangzhou, China). The pcDNA3.1 plasmid encoding full-length cDNA sequence of MTA2 (pcMTA2) and the empty vector were purchased from Biogot Biotechnology (Nanjing, China). For cell transfection, a total of $2 \times 10^{5}$ cells were plated in six-well plates. After 24 hours, miR-148b mimic or pcMTA2 or control was transfected to cells with Lipofectamine 2000 (Thermo Fisher Scientific) according to the manufacturer's protocol.

\section{Western blot analysis}

Western blot assays were performed as previously described. ${ }^{14,15}$ Briefly, 48 hours after transfection, cells were lysed with radioimmunoprecipitation assay buffer (Thermo 
Fisher Scientific) and proteins were quantified by bicinchoninic acid method. Proteins were then separated by $10 \%$ sodium dodecyl sulfate polyacrylamide gel electrophoresis (SDS-PAGE) and transferred to polyvinylidene difluoride membranes. The membranes were incubated with 5\% nonfat milk and probed with the antibodies against $\beta$-actin $(1: 10,000$ diluted; Santa Cruz Biotechnology Inc., Dallas, TX, USA) or MTA2 (1:2,000 diluted; Santa Cruz Biotechnology), followed by horseradish peroxidase (HRP)-labeled secondary antibodies. The signals were detected by the enhanced chemiluminescence detection system (Pierce, Rockford, IL, USA). The protein levels of MTA2 were normalized to $\beta$-actin. Three independent experiments were performed.

\section{Cell Counting Kit-8 (CCK8) cell counting assay}

Cells transfected with miR-148b mimic or miR-Ctrl were seeded in 96-well plates at $5 \times 10^{3}$ cells/well. At 24 and 48 hours after plating, $20 \mu \mathrm{L}$ of CCK-8 solutions (Beyotime, Shanghai, China) were added. After incubation for 2 hours, the absorbance at $450 \mathrm{~nm}$ was measured with a microplate reader. Three independent experiments were conducted with six replicates in each experiment.

\section{Colony formation assay}

Cells were seeded in six-well plates. miR-148b mimic or miRCtrl was transfected to cells and cultured for 48 hours. The cells were then washed three times with PBS and cultured in DMEM containing $10 \%$ FBS for 2 weeks in an incubator. The colonies were fixed with methanol and stained with crystal violet. The number of colonies was counted under an inverse microscope. Three independent experiments were performed.

\section{Transwell migration and invasion assays}

For transwell migration assays, $5 \times 10^{4}$ cells transfected with miR-148b mimic or miR-Ctrl were suspended in $100 \mu \mathrm{L}$ of serum-free DMEM and seeded in the upper chamber of transwell inserts (with $8.0 \mu \mathrm{m}$ pores; Corning Inc., Corning, NY, USA). For invasion assays, the upper chambers were pre-coated with Matrigel (BD Biosciences, San Jose, CA, USA). A total of $500 \mu \mathrm{L}$ of complete medium (DMEM with $10 \%$ FBS) was loaded to the lower chamber as a chemoattractant. After 20 hours of incubation, the migrated or invaded cells in the lower surfaces of the membranes were fixed with methanol and stained with crystal violet. Cells were observed with a light microscope and counted in five randomly selected fields. Three independent experiments were performed.

\section{Luciferase assay}

A total of 40,000 cells of CNE2 and C666-1 were cultured in 24-well plates and incubated for 24 hours before transfection. The pGL3-MTA2 3'UTR-wild type (WT) plasmid or pGL3-MTA2 3'UTR-mutant (Mut) plasmid together with the control vector pRL-TK (Promega Corporation, Fitchburg, WI, USA), and miR-148b mimic or miR-Ctrl, were cotransfected to cells with Lipofectamine 2000 (Thermo Fisher Scientific). After incubation for 48 hours, the cells were harvested, and the firefly and renilla luciferase activities were determined by the dual luciferase assay (Promega Corporation). Renilla luciferase activity was used for normalization. Three independent experiments were performed.

\section{Statistical analysis}

Results are presented as mean \pm standard deviation of at least three independent experiments and analyzed by SPSS 13.0 software (SPSS Inc., Chicago, IL, USA). Student's $t$-test or one-way analysis of variance with least significant difference (LSD) test for multiple comparisons was performed for comparison between groups. Pearson's correlation assay was used to analyze the correlation. Differences were regarded to be statistically significant at $P<0.05$.

\section{Results}

\section{MiR-I48b is downregulated in NPC tissues and cell lines}

To investigate the exact function of miR-148b in NPC, we detected the expression of miR-148b by qRT-PCR in 20 cases of noncancerous nasopharyngeal tissues and 25 cases of NPC tissues. MiR-148b expression was greatly decreased in NPC tissues compared with nasopharyngeal tissues (Figure 1A). Meanwhile, the expression of miR-148b was downregulated in NPC cell lines (CNE1, CNE2, C666-1 and HNE1) compared with nasopharyngeal epithelium cell, NP69 (Figure 1B). Among the cell lines detected, C666-1 and CNE2 cells had the lowest miR-148b expression so that they were chosen for the subsequent gain of function experiment.

\section{Ectopic miR-148b expression suppressed proliferation, migration, and invasion in NPC cells}

To explore the functional role of miR-148b in NPC, miR$148 \mathrm{~b}$ mimic or mimic control (miR-Ctrl) was transfected to C666-1 and CNE2 cells. As shown in Figure 2A, miR$148 \mathrm{~b}$ mimic transfection dramatically increased miR-148b expression in CNE2 and C666-1 cells. Colony formation assays demonstrated that miR-148b transfection resulted 
A

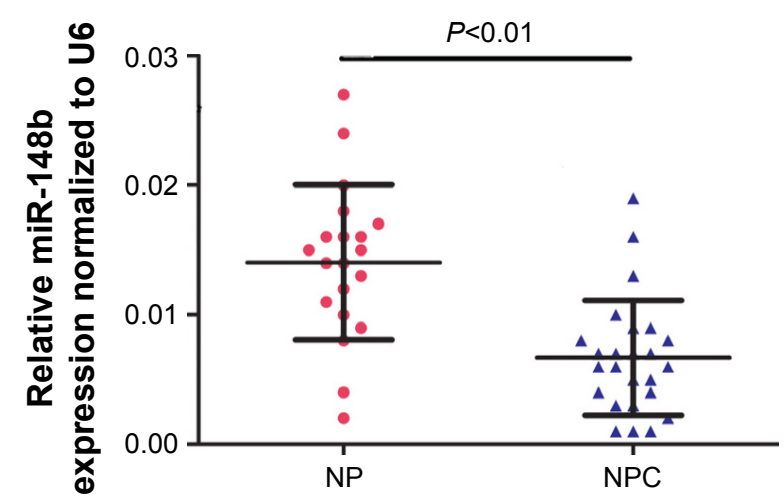

B

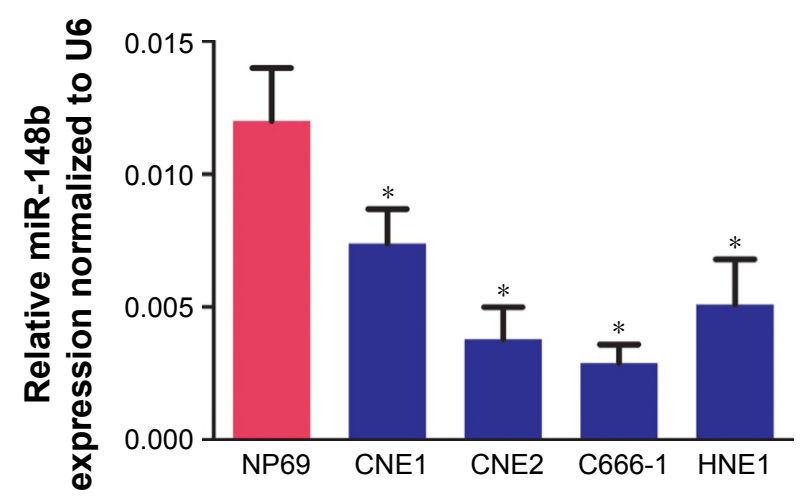

Figure I Downregulation of miR-I48b in NPC tissues and cell lines.

Notes: (A) Expression of miR- I48b in 20 cases of noncancerous NP tissues and 25 cases of NPC tissues was detected by qRT-PCR. (B) Expression of miR-I48b in NPC cell lines (CNEI, CNE2, C666-I and HNEI) and nasopharyngeal epithelium cell, NP69, was detected by qRT-PCR (*P<0.05 vs NP69).

Abbreviations: miR, microRNA; NP, nasopharyngeal; NPC, nasopharyngeal carcinoma; qRT-PCR, quantitative reverse transcription polymerase chain reaction; U6, U6 snRNA.

in the formation of fewer and smaller colonies, compared with control group (Figure 2B). CCK-8 assays demonstrated that miR-148b inhibited the growth of NPC cells in vitro, especially in C666-1 cell (Figure 2C). Transwell assays revealed that miR-148b transfection significantly inhibited the migration and invasion of CNE2 and C666-1 cells, as shown in Figure 2D. These results showed that miR-148b could inhibit the growth, migration, and invasion of NPC cells in vitro.

\section{MTA2 is a direct target of miR-I48b}

We had previously reported that MTA2 promoted proliferation and metastasis of NPC cells and acted as an oncogene in NPC. ${ }^{13}$ Using a miRNA targeting prediction program TargetScan (http://www.targetscan.org), we found that the 3'UTR of MTA2 mRNA had a complementary sequence for the seed region of miR-148b (Figure 3A), suggesting that MTA2 is a potential target of miR-148b. To verify whether MTA2 was a true target of miR-148b, we conducted luciferase assays in CNE2 and C666-1 cells. We found that, compared with control, miR-148b mimic transfection inhibited the luciferase activity when cells were co-transfected with WT 3'UTR of MTA2. However, no differences in luciferase activity between miR-148b mimic and control group were found when cells were co-transfected with Mut type 3'UTR of MTA2 (Figure 3B). Western blot analysis revealed that miR-148b remarkably downregulated MTA2 protein expression in CNE2 and C666-1 cells (Figure 3C). Notably, we found that the mRNA level of MTA2 was negatively related to miR-148b level in NPC tissues $(r=-0.677$, $P<0.01$, Figure 3D). Overall, these experiments identified that MTA2 was a direct target of miR-148b.

\section{The tumor suppressive effects of miR-I48b on C666-I cell are partially reversed by MTA2 restoration}

To verify whether the tumor suppressive role of miR-148b in NPC cells was mediated by MTA2, the expression of MTA2 was rescued in miR-148b-overexpressing cells (Figure 4A). The highly metastatic C666-1 cell, which was more sensitive to miR-148b treatment demonstrated in CCK-8 and colony formation assays, was used in the rescue experiment. We found that the restoration of MTA2 expression in C666- 1 cell partially reversed the inhibitive effects on cell proliferation, migration, and invasion induced by miR-148b overexpression (Figure 4B-D).

\section{Discussion}

Dysregulated expression of miRNAs had been shown to play important roles in regulating biological behaviors of NPC. ${ }^{16}$ The tumor-suppressive miR-29c, which was frequently reduced in NPC tissues, suppressed NPC progression and metastasis by targeting multiple genes, including TIAM1, ${ }^{17}$ Mcl-1 and Bcl-2. ${ }^{18}$ Moreover, miR-29c increased the sensitivities of NPC to chemotherapy and radiotherapy. ${ }^{18}$ The oncogenic miR-155 was upregulated in NPC by LMP1 and LMP2A, two proteins encoded by Epstein-Barr virus. ${ }^{19,20}$ miR-155 promoted the malignant biological behaviors of NPC cells by targeting JMJD1 ${ }^{20}$ and ZDHHC2 ${ }^{21}$

MiR-148b, as a member of the miR-148/152 family, plays important roles in development and diseases. ${ }^{22} \mathrm{MiR}-$ $148 \mathrm{~b}$ had been reported to be downregulated in some human cancers. ${ }^{7,8,23}$ Dysregulated miR-148b in pancreatic cancer was correlated with increased tumor size, advanced clinical stage, 
A

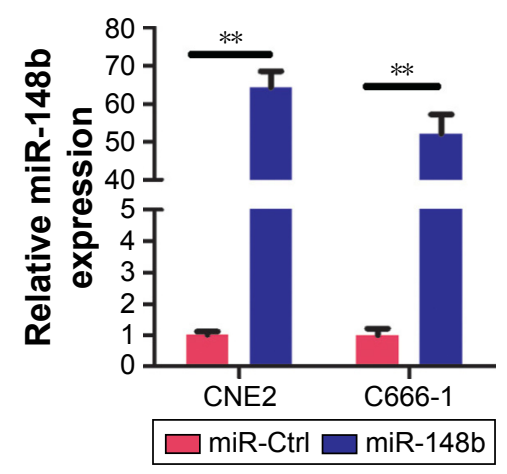

C
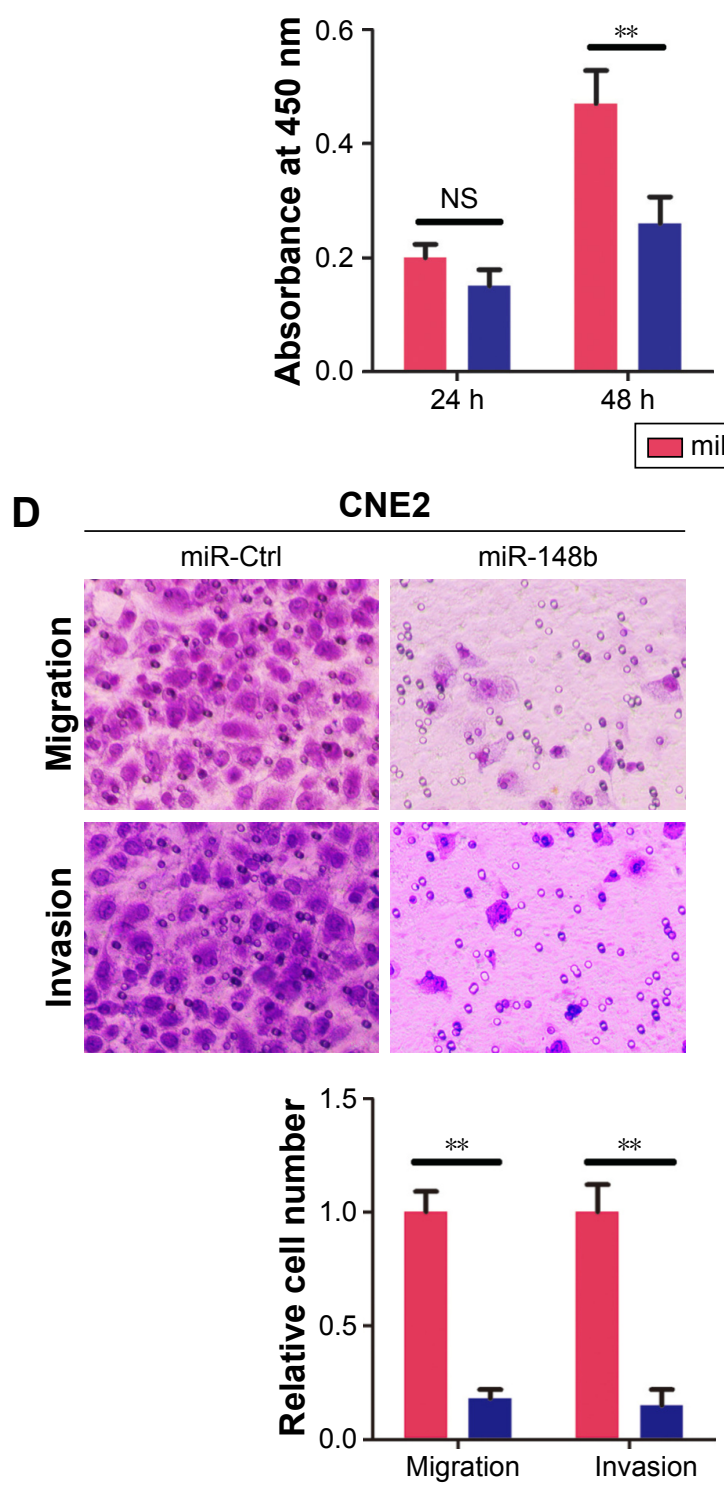

B miR-Ctrl miR-148b

CNE2

C666-1
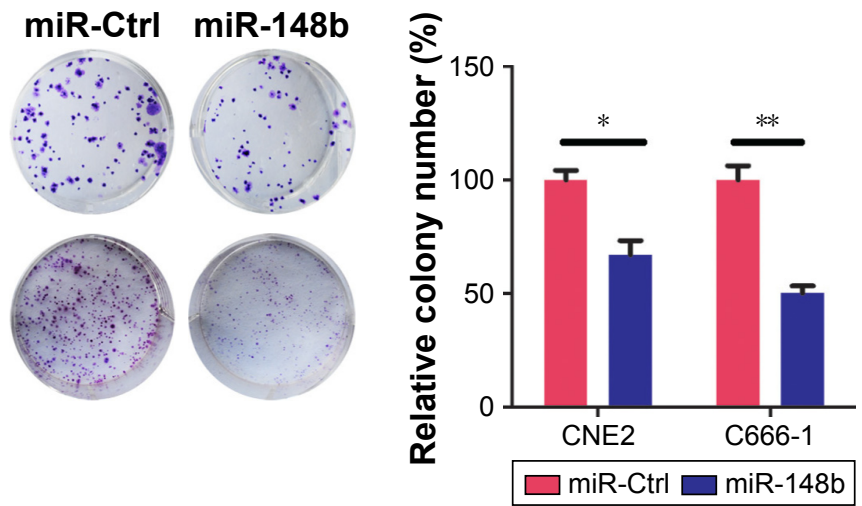

C666-1
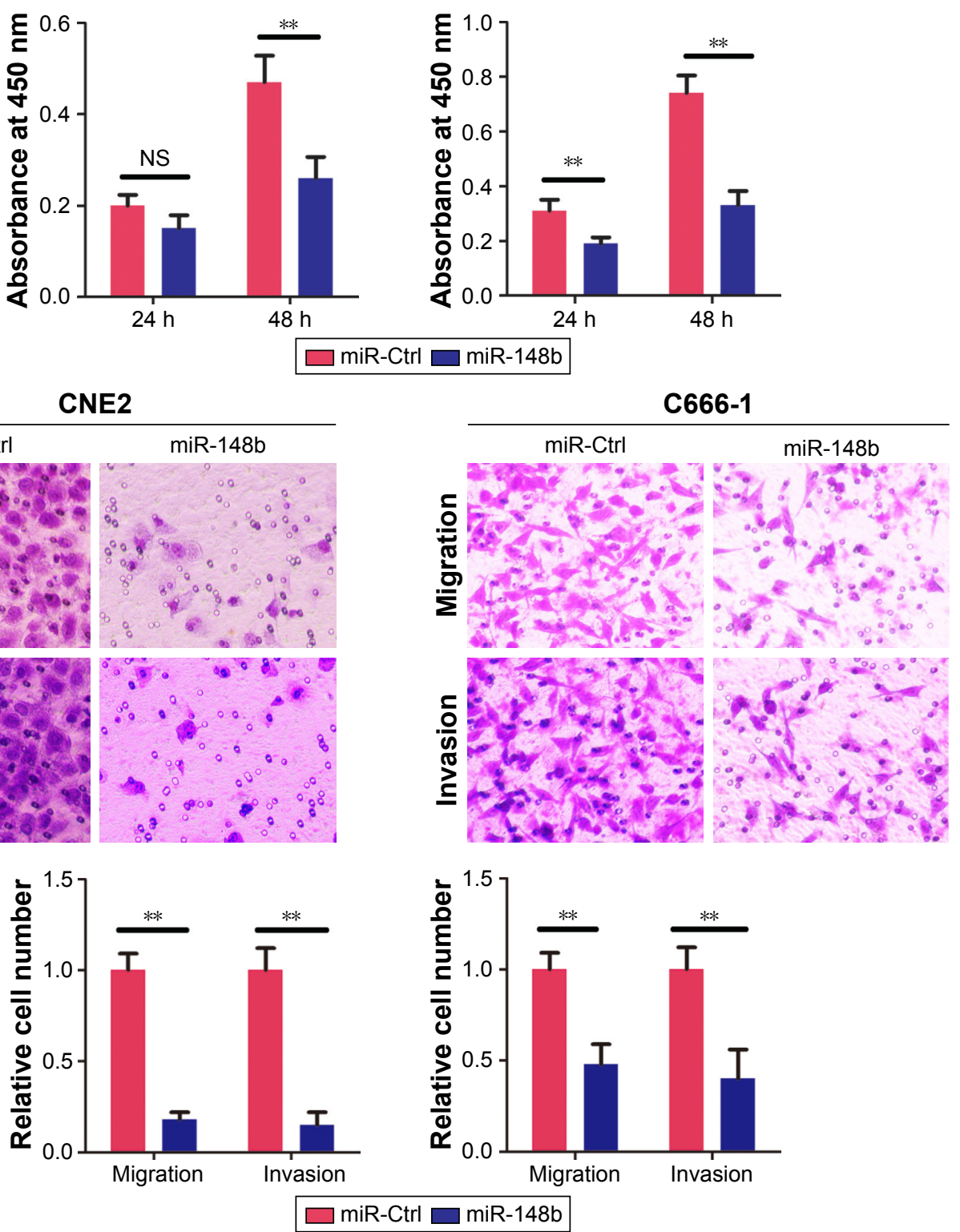

Figure 2 Inhibition effects of miR-I48b on proliferation, migration, and invasion of CNE2 and C666-I cells.

Notes: (A) Forty-eight hours after transfection with miR-I48b mimic or miR-Ctrl. The miR-I48b expression in CNE2 and C666-I cells was determined by qRT-PCR. Colony formation assays (B) and CCK-8 assays (C) were conducted to evaluate the effects of miR-148b overexpression on proliferation of CNE2 and C666-I cells. (D) Transwell assays were conducted to evaluate the effects of miR-I48b on migration and invasion of CNE2 and C666-I cells ( $* P<0.05$, $* * P<0.0 \mathrm{I})$.

Abbreviations: CCK-8, Cell Counting Kit-8; Ctrl, control; h, hours; miR, microRNA; NS, no significance; qRT-PCR, quantitative reverse transcription polymerase chain reaction. 


\section{A}

B

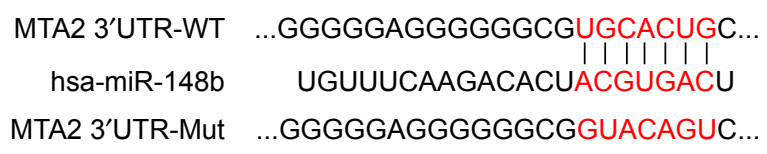

hsa-miR-148b

UGUUUCAAGACACUACGUGACU

MTA2 3'UTR-Mut ...GGGGGAGGGGGGCGGUACAGUC...

CNE2

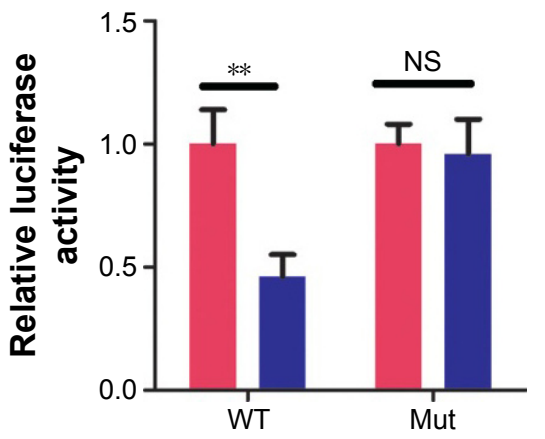

C666-1

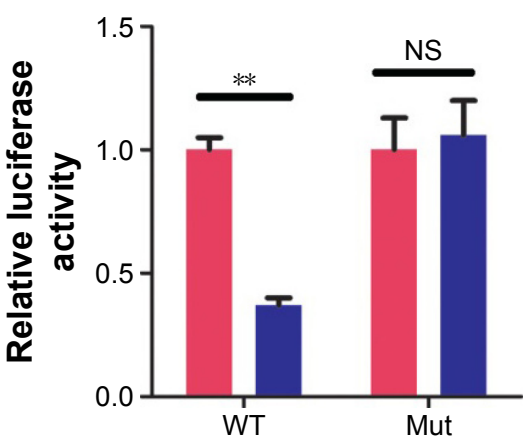

C
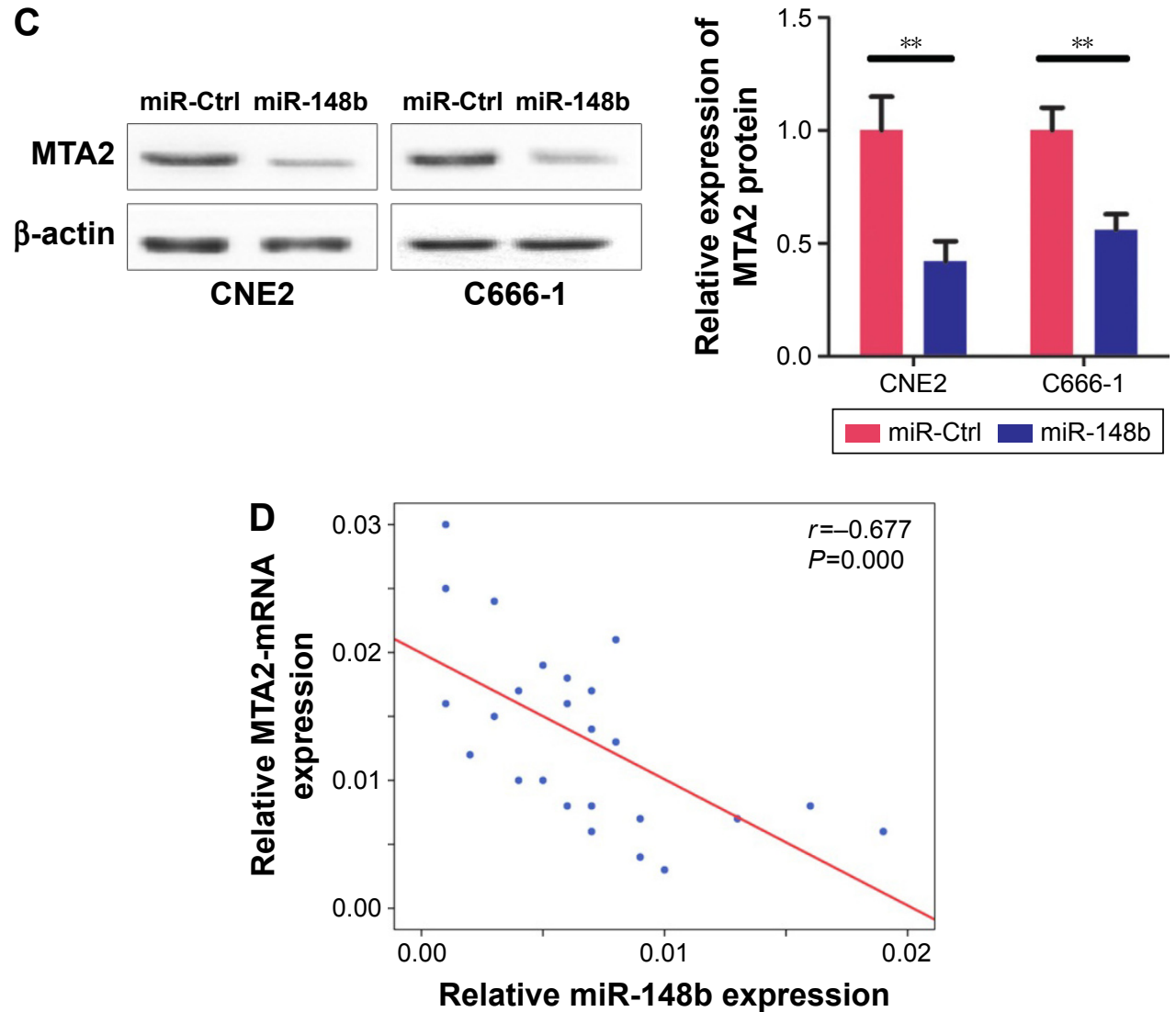

Figure 3 MTA2 is a target of miR-148b.

Notes: (A) The binding site of miR-148b in the 395-40I bp position in the 3'UTR of MTA2 mRNA predicted by TargetScan. (B) The dual luciferase reporter activity analysis of WT and Mut type MTA2 3'UTR reporter constructs after co-transfection with miR-I48b or miR-Ctrl. (C) Ectopic miR-I48b expression reduced the protein expression of MTA2 in CNE2 and C666-I cells. (D) The correlation between miR-I48b and MTA2 mRNA expression in 25 cases of NPC tissue was analyzed by Pearson's correlation assay $(* * P<0.01)$.

Abbreviations: Ctrl, control; miR, microRNA; MTA2, metastasis-associated gene 2; Mut, mutant; NPC, nasopharyngeal carcinoma; NS, no significance; WT, wild type; UTR, untranslated region.

distant metastasis and poor prognosis. Ectopic miR-148b expression inhibited the growth and metastasis of pancreatic cancer cells by targeting AMPK $\alpha 1 .{ }^{8}$ In hepatocellular carcinoma, miR-148b negatively regulated WNT1/ $\beta$-catenin pathway by targeting WNT. ${ }^{19}$ Moreover, miR-148b was critical in the maintenance of properties of cancer stem cells by targeting Neuropilin-1 in hepatocellular carcinoma, suggesting that the restoration of miR-148b may be a new 
A

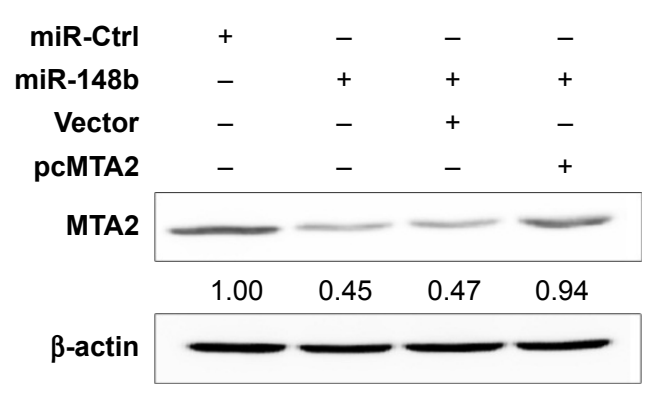

C

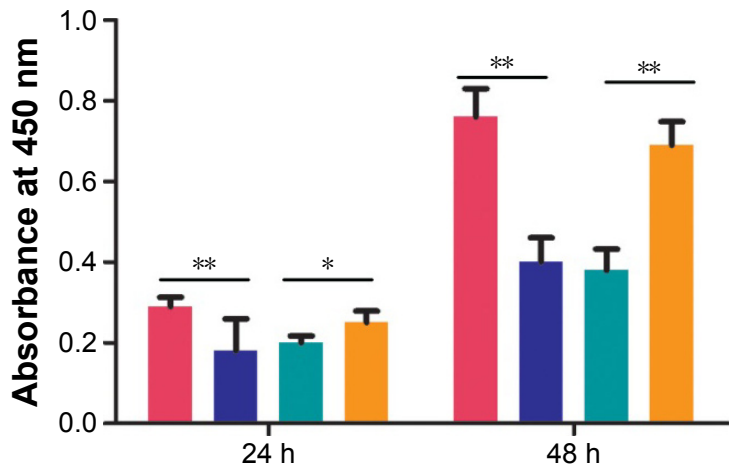

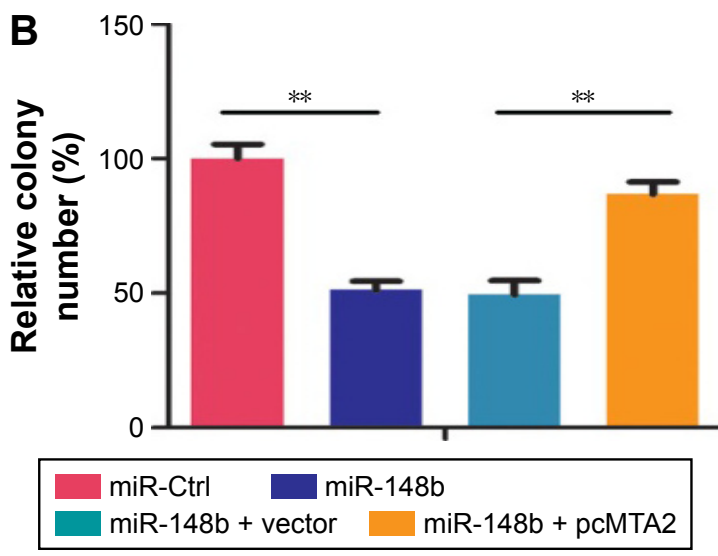

D

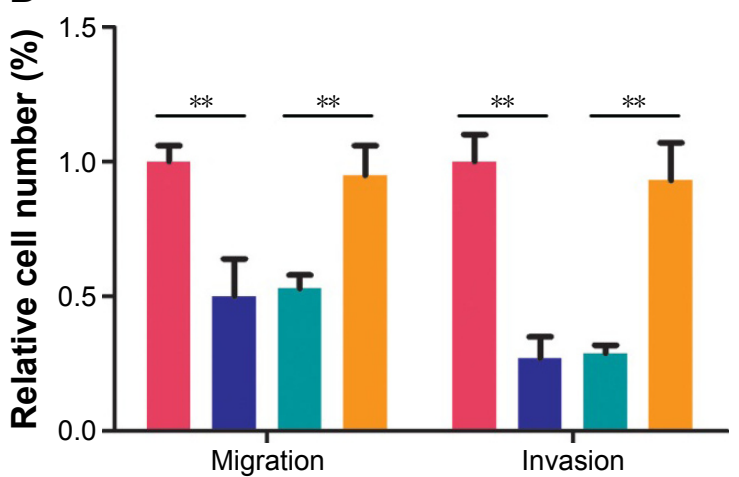

miR-Ctrl miR-148b miR-148b + vector miR-148b + pcMTA2

Figure 4 The tumor-suppression effects of miR-I48b on C666-I cell were partially reversed by MTA2 restoration.

Notes: (A) C666-I cells were transfected with the empty vector or MTA2 expression vector in miR-I48b-overexpressing C666-I cells, and MTA2 protein expression was analyzed by Western blot. The effects of MTA2 restoration on cell growth were detected by colony formation assays (B) and CCK-8 assays (C). (D) The effects of MTA2 restoration on cell migration and invasion were detected by transwell assays $(* P<0.05, * * P<0.01)$.

Abbreviations: CCK-8, Cell Counting Kit-8; Ctrl, control; miR, microRNA; h, hours; MTA2, metastasis-associated gene 2; pcMTA2, MTA2 expression plasmid pcDNA3.I.

therapeutic strategy by targeting cancer stem cells. ${ }^{24} \mathrm{miR}$ $148 \mathrm{~b}$ inhibits malignant behaviors of glioma cells through targeting HOTAIR. ${ }^{7}$ These studies, overall, revealed that miR-148b may play important roles in NPC. However, the functions of miR-148b in NPC are still largely unknown.

In the current study, we found that miR-148b expression was frequently decreased in NPC tissues and cell lines. In the NPC cell lines detected, the highly metastatic C666-1 cell had the lowest miR-148b expression, suggesting that miR-148b may be related to the metastasis of NPC. Besides C666-1 cell, another NPC cell line, CNE2, with relatively low miR-148b expression, were transfected with miR-148b mimic for the gain of function study. The results showed that the overexpression of miR-148b significantly inhibited proliferation, colony formation, migration, and invasion of C666-1 and CNE2 cells. These experiments revealed that miR-148b acts as a tumor suppressor in NPC.

MTA2 had been reported to promote tumor progression. ${ }^{25}$ MTA2 overexpression promoted the proliferation and invasion of gastric cancer cells through stimulating the secretion of interleukin $11 .{ }^{26}$ MTA2 promoted the migration of breast cancer cells by activating the RhoA signaling pathway. ${ }^{27}$ We had previously found that MTA2 was upregulated in NPC. MTA2 promoted the growth and metastasis of NPC cells in vitro and in vivo. ${ }^{13}$ In silico analysis by TargetScan reveals that MTA2 is a potential target of miR-148b. Subsequently, the luciferase assays were performed and MTA2 was identified to be a novel target of miR-148b. In addition, we found that the mRNA level of MTA2 was negatively related to miR-148b in NPC tissues, indicating that miR-148b can negatively regulate MTA2 expression in NPC. The rescue experiment was performed to explore the functional role of miR-148b/MTA2 axis in C666-1 cell. We found that the tumor-suppressive effects of miR-148b were partially reversed. The results indicated that reduced miR-148b expression promoted proliferation, migration, and invasion of NPC cells by targeting MTA2. The current in vitro study reveals the function of miR-148b 
in NPC. However, further investigation is needed to fully understand the role of miR-148b in NPC, such as in vivo study and exploring the interaction between miR-148b and its potential targets in NPC.

\section{Conclusion}

Our study demonstrates that miR-148b acts as a tumor suppressor in NPC. MTA2 is a novel target of miR-148b. MiR-148b inhibits malignant behaviors of NPC cells, at least partly, through the repression of MTA2 expression.

\section{Acknowledgments}

This study was supported by the Medical Scientific Research Foundation of Guangdong Province, China (grants A2016529 and A2015105), Administration of Traditional Chinese Medicine of Guangdong Province, China (grant A20171148), National Natural Science Foundation of China (grant 81201763), National Natural Science Foundation of Guangdong, China (grant 2016A030310357) and the Science Research Foundation of Guangdong Medical University, China (grant M2015011).

\section{Disclosure}

The authors report no conflicts of interest in this work.

\section{References}

1. Carioli G, Negri E, Kawakita D, Garavello W, La Vecchia C, Malvezzi M. Global trends in nasopharyngeal cancer mortality since 1970 and predictions for 2020: focus on low-risk areas. Int J Cancer. 2017; 140(10):2256-2264.

2. Yoshizaki T, Ito M, Murono S, Wakisaka N, Kondo S, Endo K. Current understanding and management of nasopharyngeal carcinoma. Auris Nasus Larynx. 2012;39(2):137-144.

3. Bartel DP. MicroRNAs: genomics, biogenesis, mechanism, and function. Cell. 2004;116(2):281-297.

4. Aleckovic M, Kang Y. Regulation of cancer metastasis by cell-free miRNAs. Biochim Biophys Acta. 2015;1855(1):24-42.

5. Tan G, Tang X, Tang F. The role of microRNAs in nasopharyngeal carcinoma. Tumour Biol. 2015;36(1):69-79.

6. Liu GL, Liu X, Lv XB, Wang XP, Fang XS, Sang Y. miR-148b functions as a tumor suppressor in non-small cell lung cancer by targeting carcinoembryonic antigen (CEA). Int J Clin Exp Med. 2014;7(8):1990-1999.

7. Wang G, Li Z, Tian N, et al. miR-148b-3p inhibits malignant biological behaviors of human glioma cells induced by high HOTAIR expression. Oncol Lett. 2016;12(2):879-886.

8. Zhao G, Zhang JG, Liu Y, et al. miR-148b functions as a tumor suppressor in pancreatic cancer by targeting AMPKalpha1. Mol Cancer Ther. 2013; 12(1):83-93.

OncoTargets and Therapy

\section{Publish your work in this journal}

OncoTargets and Therapy is an international, peer-reviewed, open access journal focusing on the pathological basis of all cancers, potential targets for therapy and treatment protocols employed to improve the management of cancer patients. The journal also focuses on the impact of management programs and new therapeutic agents and protocols on
9. Zhang JG, Shi Y, Hong DF, et al. MiR-148b suppresses cell proliferation and invasion in hepatocellular carcinoma by targeting WNT1/ beta-catenin pathway. Sci Rep. 2015;5:8087.

10. Chang H, Zhou X, Wang ZN, et al. Increased expression of miR-148b in ovarian carcinoma and its clinical significance. Mol Med Rep. 2012; 5(5): $1277-1280$.

11. Qian TM, Zhao LL, Wang J, et al. miR-148b-3p promotes migration of Schwann cells by targeting cullin-associated and neddylationdissociated 1. Neural Regen Res. 2016;11(6):1001-1005.

12. Covington KR, Fuqua SA. Role of MTA2 in human cancer. Cancer Metastasis Rev. 2014;33(4):921-928.

13. Wu M, Ye X, Deng X, Wu Y, Li X, Zhang L. Upregulation of metastasisassociated gene 2 promotes cell proliferation and invasion in nasopharyngeal carcinoma. Onco Targets Ther. 2016;9:1647-1656.

14. Ma L, Deng X, Wu M, Zhang G, Huang J. Down-regulation of miRNA204 by LMP- 1 enhances CDC42 activity and facilitates invasion of EBV-associated nasopharyngeal carcinoma cells. FEBS Lett. 2014; 588(9):1562-1570.

15. Deng $\mathrm{X}, \mathrm{Ma} \mathrm{L}, \mathrm{Wu} \mathrm{M}$, et al. miR-124 radiosensitizes human glioma cells by targeting CDK4. J Neurooncol. 2013;114(3):263-274.

16. Bruce JP, Liu FF. MicroRNAs in nasopharyngeal carcinoma. Chin J Cancer. 2014;33(11):539-544.

17. Liu N, Tang LL, Sun Y, et al. MiR-29c suppresses invasion and metastasis by targeting TIAM1 in nasopharyngeal carcinoma. Cancer Lett. 2013;329(2):181-188.

18. Zhang JX, Qian D, Wang FW, et al. MicroRNA-29c enhances the sensitivities of human nasopharyngeal carcinoma to cisplatin-based chemotherapy and radiotherapy. Cancer Lett. 2013;329(1):91-98.

19. Zhu X, Wang Y, Sun Y, Zheng J, Zhu D. MiR-155 up-regulation by LMP1 DNA contributes to increased nasopharyngeal carcinoma cell proliferation and migration. Eur Arch Otorhinolaryngol. 2014;271(7): 1939-1945.

20. Du ZM, Hu LF, Wang HY, et al. Upregulation of MiR-155 in nasopharyngeal carcinoma is partly driven by LMP1 and LMP2A and downregulates a negative prognostic marker JMJD1A. PLoS One. 2011;6(4):e19137.

21. Jiang YX, Du ZM, Jiao L, et al. Inhibition of MiR-155 suppresses cell migration in nasopharyngeal carcinoma through targeting ZDHHC2. Int J Clin Exp Med. 2015;8(6):8472-8484.

22. Chen Y, Song YX, Wang ZN. The microRNA-148/152 family: multifaceted players. Mol Cancer. 2013;12:43.

23. Liao YH, Chang YH, Sung LY, et al. Osteogenic differentiation of adipose-derived stem cells and calvarial defect repair using baculovirusmediated co-expression of BMP-2 and miR-148b. Biomaterials. 2014; 35(18):4901-4910.

24. Liu Q, Xu Y, Wei S, et al. miRNA-148b suppresses hepatic cancer stem cell by targeting neuropilin-1. Biosci Rep. 2015;35(4):e00229.

25. Toh Y, Nicolson GL. The role of the MTA family and their encoded proteins in human cancers: molecular functions and clinical implications. Clin Exp Metastasis. 2009;26(3):215-227.

26. Zhou C, Ji J, Cai Q, et al. MTA2 enhances colony formation and tumor growth of gastric cancer cells through IL-11. BMC Cancer. 2015; $15: 343$.

27. Covington KR, Brusco L, Barone I, et al. Metastasis tumor-associated protein 2 enhances metastatic behavior and is associated with poor outcomes in estrogen receptor-negative. Breast Cancer Res Treat. 2013; 141(3):375-384.

\section{Dovepress}

patient perspectives such as quality of life, adherence and satisfaction The manuscript management system is completely online and includes a very quick and fair peer-review system, which is all easy to use. Visit http://www.dovepress.com/testimonials.php to read real quotes from published authors. 